Review Article

\title{
Transportation Course of Macromolecules to the Nucleus from the Extracellular Environment: Steroid Hormones' Cellular Entry Mode Revisited
}

\author{
Toshikazu Nishimura \\ Japan College of Rehabilitation and Welfare Professionals, Nagoya, Japan \\ Email address: \\ to4nishimura@yahoo.co.jp
}

To cite this article:

Toshikazu Nishimura. Transportation Course of Macromolecules to the Nucleus from the Extracellular Environment: Steroid Hormones' Cellular Entry Mode Revisited. Cell Biology. Vol. 6, No. 1, 2018, pp. 9-12. doi: 10.11648/j.cb.20180601.12

Received: March 15, 2018; Accepted: April 8, 2018; Published: May 5, 2018

\begin{abstract}
Polyomavirus virions such as simian virus 40 (SV40), antinuclear antibodies such as immunoglobulin G (IgG) and steroid hormones all enter the nucleus from the extracellular environment. Testosterone-bovine serum albumin conjugate labeled with $2 \mathrm{~nm}$ colloidal gold (testosterone-BSA-gold) is taken up by endocytosis into target cells, and enter the nucleus through a similar route as SV40 nuclear migration. Upon injection into the vascular system of rats, IgG coupled with hydrocortisone also enters the hormone-target cell nuclei with intact antigenicity. These results suggest that steroid hormones could act as transporters to deliver exogenous macromolecules, e.g. drugs, into their target cell nuclei in vivo, although further studies are required on whether steroid hormones coupled with proteins exert genomic actions in the nucleus, etc. Finally, testosterone-BSA-gold seems to be isolated from the cytosol in the processes of nuclear entry. Together, these findings challenge the popular belief that steroid hormones mostly enter the cell in unbound form via uncontrolled passive diffusion.
\end{abstract}

Keywords: Vesicular Trafficking, Macromolecules, Transportation Course, Nuclear Diaphragm, Target Cell Nuclei, Exterior of Cells, Steroid-Protein Conjugates, Intact Antigenicity

\section{Introduction}

Various large molecules destined for cell nucleus arrive from the extracellular environment. Many of the mechanisms by which such molecules enter the cell and its nucleus are still unclear. For example, antinuclear antibodies can enter the nucleus from the exterior of a cell. However, native IgG does not pass freely the cell- or nuclear membrane; IgG introduced to the cytoplasm did not enter the nucleus $[1,2]$. Polyomavirus virions such as simian virus 40 (SV40) comprise proteins and DNA, and are able to enter the nucleus from the extracellular environment [3]. Substances such as SV40 tumor antigen and nuclear proteins migrate into the nucleus from the cytoplasm $[4,5]$. IgG with synthetic peptides containing nuclear localization signal such as that of SV40 tumor antigen could enter the nucleus from the cytoplasm by active transport through nuclear pore complexes (NPCs) $[2,6]$. This review reports about vesicular trafficking of macromolecules to the nucleus from extracellular environment.

\section{Transportation Course of Macromolecules to the Nucleus from the Extracellular Environment}

In some infectious processes, virus-containing vesicles fuse with the outer nuclear membrane, delivering the virus particles into the perinuclear cisterna (Figure 1a) [7, 8]. In our search for other entryways to the nucleus, migration of SV40 was pursued in cultured cells, using ferritin and concanavalin A as cell membrane markers. Ferritin particles introduced into the cytoplasm did not enter the nucleus by themselves. In contrast, SV40-containing vesicles with ferritin particles were observed close to a single-bilayer nuclear membrane or a diaphragm (Figure 1b, c) $[9,10]$. The nucleoplasmic side of the diaphragm was covered with electron-dense materials, and cell membrane markers were localized along the 
nucleoplasmic side of the inner nuclear membrane $[9,10]$. These results suggest that SV40-containing vesicle membrane fuses to a single-bilayer diaphragm in the nuclear envelope in order to transport virus particles into the nucleoplasm, and that the exogenous macromolecules used here as cell membrane markers were transported into the nucleus in this manner (Figure 1b) [9].

\section{Diaphragms in Nuclear Envelope}

A question then arose whether diaphragms in nuclear envelope are formed by hemifusion between the outer- and inner nuclear membrane, or by deletion of the outer membrane. Complete fusion of two membranes such as pore formation occurs through the process of hemifusion (Figure 1d) [11]. The hemifused area then enlarges [12]. To validate the presence of pores other than NPCs in the nuclear envelope, subacrosomal nuclear envelopes (SNEs) of spermatids were observed under electron microscope, as SNE is devoid of NPCs. Two membranes of SNE are in close apposition. The continuity between the outer and inner nuclear membranes of SNE was observed successfully [13], indicating the possibility that there is hemifusion membrane in the SNE. Earlier, we found pores that are different from NPCs in nuclear export of baculovirus nucleocapsids. Recombinant baculovirus nucleocapsids (45 nm x 280-300 nm) are formed in the nucleoplasm, and migrate into the cytoplasm to bud through cell membrane. In the study using rapid cryofixation, we proposed that the nucleocapsids pass through small pores formed in the protrusion of double membranes derived from the nuclear envelope [14]. From our study on the nuclear entry of testosterone-BSA-gold discussed below, the diaphragm seems to be formed by hemifusion of both membranes.

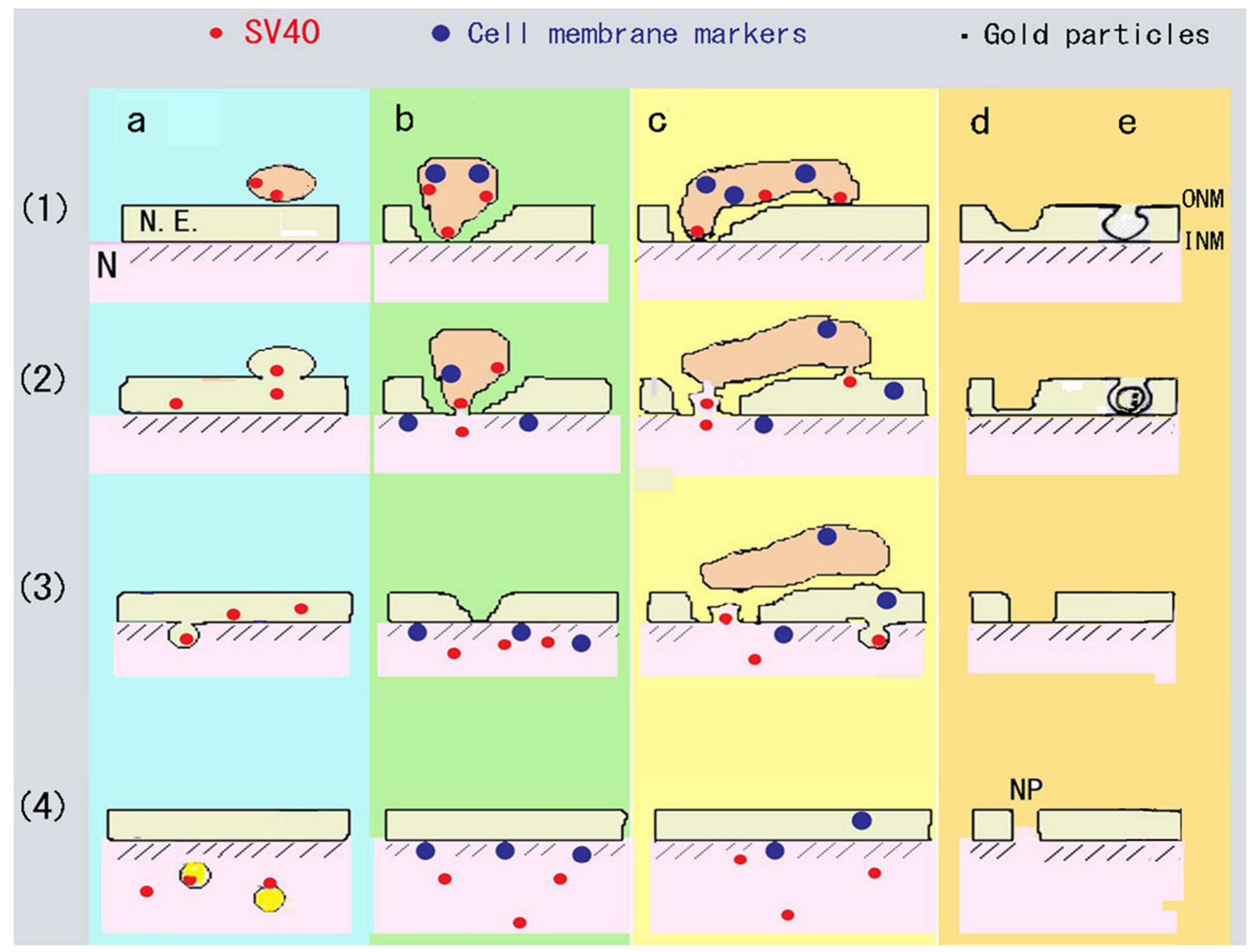

Figure 1. Schema of fusion between nuclear membranes and vacuole membrane.

a. Nuclear entry of SV40 proposed by Maul. $(1,2)$ Vacuoles containing SV40 fuse with outer nuclear membrane. (2) Virus particles enter perinuclear cisterna. (3) Virus particles are enveloped by inner nuclear membrane. (4) Membrane envelopes free SV40 in the nucleus.

b. (1) Vacuoles containing cell membrane markers and SV40 are located close to single membrane-like envelopes, or diaphragms. (2) Fusion between vacuoles' membrane and diaphragms. $(3,4)$ Cell membrane markers and SV40 enter the nucleus.

c. (1) Large vacuoles containing cell membrane markers and SV40 are located near the nucleus. (2) Parts of vacuoles' membrane fuse with diaphragms, while other parts fuse with the outer nuclear membrane. $(3,4)$ Cell membrane markers and SV40 enter the nucleus, and some markers remain in the perinuclear cisterna.

d. $(1,2)$ Invagination of the outer nuclear membrane toward the inner nuclear membrane. (3) Formation of hemifusion diaphragm. (4) Formation of pore. e. (1) Invagination of the outer nuclear membrane of rat spermatogenic cells where testosterone-BSA-gold was injected. (2) A double-membrane-like vesicle with testosterone-BSA-gold in the nuclear envelope.

$\mathrm{N}$ : nucleus; NE: nuclear envelope; NP: nuclear pore; ONM: outer nuclear membrane; INM: inner nuclear membrane. 


\section{Steroid Hormones as Carriers to Deliver Exogenous Proteins into the Target Cell Nuclei}

Steroid hormones circulate in blood plasma in three different forms: albumin-bound, steroid hormone-binding globulin (SHBG)-bound, and free [15]. In the classical model of genomic steroid hormone action, lipophilic hormones are first released from their carrier proteins and cross the cell membrane by passive diffusion in their free form $[16,17]$. Contrary to this belief, SHBG coupled with $\left[{ }^{3} \mathrm{H}\right]$-testosterone (testosterone-SHBG) is internalized by receptor-mediated endocytosis in spermatogenic cells, which are target cells of testosterone, and then enters their nuclei in vitro $[18,19]$. Colloidal gold embedded in epoxy resin becomes visible as silver deposits on the sections after silver enhancement [20]. Upon injection into the vascular system of rats, testosterone-bovine serum albumin conjugate labeled with 2 $\mathrm{nm}$ colloidal gold (testosterone-BSA-gold) is taken up by endocytosis into the target cells of testosterones such as round spermatids, and then enters the nucleoplasm [10, 20, 21]. In contrast, the nuclei of cells that are not targeted by testosterone such as thymocytes and hepatocytes showed very few silver deposits implying the presence of testosterone-BSA-gold [20]. These results suggest that the nuclear entry of testosterone-BSA-gold is specific to the target cells of testosterone. From the distribution of silver deposits, it has become clear that hydrocortisone-BSA-gold conjugates injected into rats enter the target cell nuclei such as hepatocytes and thymocytes [22]. The target-specificity suggests that the fate of gold labeled-steroid-BSAs may be decided at the cell membrane level.

In the spermatogenic cells of rat injected with testosterone-BSA-gold, the silver deposits were present on the cell membrane, vesicles, Golgi region, acrosome, subacrosomal space, both the post-acrosomal and the subacrosomal nuclear envelope, and the nucleoplasm [10,21]. In observations without silver enhancement, a single-bilayer nuclear membrane or a diaphragm was visible in the SNE [10, 13]. In post-acrosomal nuclear envelope, the outer nuclear membrane was invaginated toward the inner nuclear membrane, and was likely to interact with the latter (Figure 1e) $[10,21]$. Furthermore, a double-membrane-like vesicle containing gold particles was observed in the pit formed by the invagination of the outer nuclear membrane (Figure 1e) $[10,21]$. These results suggest that testosterone-BSA-gold is transported by vesicles from the extracellular environment to the nucleoplasm. This route resembles the entryway proposed for nuclear migration of SV40 (Figure 1b) [21].

We also investigated whether BSA in the steroid-BSAs remains intact in the cell nuclei. For this purpose, testosterone-BSA, hydrocortisone-BSA or corticosterone-BSA was injected into rats; it showed that BSA-steroid hormone conjugates enter the hormone-target cell nuclei while maintaining antigenicity [23]. Steroid-BSA binds to nuclear receptors $[24,25]$. Bovine IgG coupled with hydrocortisone injected into adrenalectomized rat vascular system enters the hormone-target cell nuclei in the liver, maintaining the antigenicity [26]. Together, these findings support the idea that steroid hormones could be useful as target cell-specific carriers to deliver exogenous macromolecules into cell nuclei.

\section{Conclusion}

Cells have various mechanisms to control the substances and their quantity that can pass through the cell- and nuclear membrane, and countermeasures have been developed by viruses such as SV40. The popular belief that lipophilic molecules such as steroid hormones can simply diffuse into cells uncontrolled seems to go against this controlling nature. Moreover, why do steroid hormones not remain in the lipid layer of biomembrane like their precursor cholesterol, if they enter cells by simple diffusion? Mounting evidences such as those mentioned above and others indicate that the passage of steroids into cells and their nuclei is better regulated than previously believed. In addition, the notion that steroids only traverse the cell membrane in unbound form is also challenged In the process of nuclear entry, steroid-BSAs seem to be isolated from the cytosol. It is unknown whether steroid hormones coupled with proteins exert genomic actions in the nuclei. Further studies are required to elucidate these processes.

\section{References}

[1] Furusawa M, Yamaizumi M, Nishimura T, Uchida T, Okada Y. Use of erythrocyte ghosts for injection of substances into animal cells by cell fusion. Methods Cell Biol; 14: 73-80.

[2] Lanford RE, Kanda P, Kennedy RC. Induction of nuclear transport with a synthetic peptide homologous to the SV40 T antigen transport signal. Cell 1986; 46; 575-82.

[3] Hummeler K, Tomassini N, Sokol F. Morphological aspects of the uptake of simian virus 40 by permissive cells. J Virol 1970; 6: 87-93.

[4] Graessmann A, Graessmann M, Tjian R, Topp WC. Simian virus 40 small-t protein is required for loss of actin cable networks in rat cells. J Virol 1980; 33:1182-91.

[5] Yamaizumi M, Uchida T, Okada Y, Furusawa M, Mitsui H. Rapid transfer of non-histone chromosomal proteins to the nucleus of living cells. Nature 1978; 273: 782-4.

[6] Yamasaki L, Lanford RE. 6-Nuclear transport receptors: specificity amid diversity. ed. CM Feldherr. Nuclear Trafficking. Academic Press, 1992; 121-174.

[7] Maul GG, Rovera G, Vorbrodt A, Abramczuk J. Membrane fusion as a mechanism of simian virus 40 entry into different cellular compartments. J Virol 1978; 28: 936-944.

[8] Nishimura T, Kawai N, Kawai M, Notake K, Ichihara I. Fusion of SV40-induced endocytotic vacuoles with the nuclear membrane. Cell Struct Funct 1986; 11: 135-141. 
[9] Nishimura T, Kawai N, Ichihara I. Interaction of endocytotic vacuoles with the inner nuclear membrane in simian virus 40 entry into CV-1 cell nucleus. Cell Struct Funct 1991; 16: 441-445.

[10] Nishimura T. Steroid hormones as transporters to carry exogenous macromolecules into the target cell nuclei in vivo. Am J Life Sci 2015; 3: 53-57.

[11] Chernomordik LV, Kozlov MM. Membrane hemifusion: crossing a chasm in two leaps. Cell 2005; 123: 375-382.

[12] Melikyan GB, White JM, Cohen FS. GPI-anchored influenza hemagglutinin induces hemifusion to both red blood cell and planar bilayer membranes. J Cell Biol 1995; 131: 679-691.

[13] Nishimura T, Nakano T. Vesicles in the subacrosomal space and partial diaphragms in the subacrosomal nuclear envelope of round spermatids of a rat injected intravenously with gold labeled-testosterone-bovine serum albumin conjugate: vesicular trafficking from acrosome to nucleus. Okajimas Folia Anat Jpn 2002; 79: 15-23.

[14] Nishimura T, Favre D, Dürrenberger M, Michel MR, Ichihara I, Koblet H. Nuclear export of recombinant baculovirus nucleocapsids through small pore or nuclear-pore-like structure in Sf9 cells. Okajimas Folia Anat Jpn 1994; 71: 83-97.

[15] Hammond GL. Plasma steroid-binding proteins: primary gatekeepers of steroid hormone action. J Endocrinol 2016; 230: R13-25.

[16] Jensen EV and DeSombre ER. Mechanism of action of the female sex hormones. Ann Rev Biochem 1972; 41: 203- 230.

[17] Caldwell JD, Londe K, Ochs SD, Hajdu Z, Rodewald A, Gebhart VM, Jirikowski GF. Three steroid-binding globulins, their localization in the brain and nose, and what they might be doing there._Steroids 2017 in press.

[18] Gerard A, En Nya A, Egloff M, Domingo M, Degrelle H, and
Gerard H. Endocytosis of human sex steroid-binding protein in monkey germ cells. Ann NY Acad Sci 1991; 637: 258-276.

[19] Gerard H, Gerard A, En Nya A, Felden F, and Gueant JL. Spermatogenic cells do internalize Sertoli androgen-binding protein: A transmission electron microscopy autoradiographic study in the rat. Endocrinology 1994; 134: 1515- 1527.

[20] Nishimura T, Ichihara I. Nuclear concentration of gold labeled-testosterone-bovine serum albumin conjugate injected intravenously in the hormone-target cells of rat. Cell Struct Funct 1997; 22: 433-442.

[21] Nishimura T, Nakano T. Nuclear translocation of gold labeled-testosterone-bovine serum albumin conjugate through the nuclear double membranes in rat spermatids. Cell Struct Funct 1997; 22: 621-629.

[22] Nishimura T, Nakano T. Nuclear localization of gold labeled-hydrocortisone-bovine serum albumin conjugate injected intravenously into the hormone-target cells of rat. Cell Struct Funct 1999; 24: 227-35.

[23] Nishimura T, Nakano T. Immunocytochemical localization of bovine serum albumin (BSA) in the liver and testis of rats injected with testosterone-BSA, hydrocortisone-BSA or corticosterone-BSA. Cell Struct Funct 2000; 25: 161-169.

[24] Beppu K. An electron microscopic study of the steroid hormone receptor in uterine cells by the colloidal gold-labeled steroid hormone. J. Electron Microsc 1989; 38: 430-440.

[25] Okuda Y, Hashimoto K, Nakayama, M. Yamamoto H. Histochemical study on androgen receptor in canine testis by an androgen labeled with colloidal gold. J. Nara Med. Assoc 1989; 40: 625-629.

[26] Nishimura T, Nakano T. Nuclear localization of bovine immunoglobulin $\mathrm{G}(\mathrm{bIgG})$ in the liver of rats injected with hydrocortisone-bIgG conjugate intravenously. Okajimas Folia Anat Jpn 2001; 78: 107-114. 